\title{
LEFT IDEALS AND 0-PRIMITIVITY IN MATRIX NEAR-RINGS
}

\author{
by J. H. MEYER
}

(Received 1st May 1990)

\begin{abstract}
Maximal left ideals in matrix rings were studied by Stone [10]. Similar results are not necessarily valid in the general near-ring case and one of the objectives of this paper is to study these differences. Furthermore, although much is known about 2-primitivity in general matrix near-rings (Van der Walt [11]), quite the opposite is true for 0-primitivity and the other objective of this paper is to present some results on 0primitivity in matrix near-rings in certain restricted cases.
\end{abstract}

1980 Mathematics subject classification (1985 Revision): 16A76.

\section{Introduction}

Matrix near-rings were introduced in 1984 by Meldrum and Van der Walt [5]. Since then several papers $([8,12,11,13,6,2,3])$ and theses $([7,1])$ were devoted to matrix near-rings and as this field of study is still very immature, many more publications are expected to follow.

The purpose of this paper is to study 0-primitivity in matrix near-rings. A good survey on 2-primitivity in matrix near-rings over any zero-symmetric near-ring has been done by Van der Walt [11]. Some results on 0-primitivity are also contained in Abbasi, Meldrum and Meyer [2], but only for a very special class of near-rings, namely the weakly distributive d.g. near-rings. Because of some complexities, we could only manage to obtain certain results in restricted cases such as finite near-rings, or near-rings having the DCCR. It seems that a considerable amount of work still needs to be done to obtain similar results in the general zero-symmetric case.

The first section merely introduces some of the basic definitions, results and techniques in matrix near-rings which will be used in this paper. For more details the interested reader should consult [5], [7] and [1]. Section 2 deals with maximal left ideals in matrix near-rings and the connections they have (or do not have) with maximal left ideals in the base near-ring. A counter-example is given to show that the near-ring case does not always necessarily follow the same pattern as in the ring case.

The final section is devoted, for the greater part, to finite zero-symmetric near-rings and 0 -primitivity. It becomes clear from this section that in order to have a reasonable understanding of modules over matrix near-rings, it is useful if one knows whether or not such modules can be embedded into a direct sum of finitely many copies of the additive group of the base near-ring. 


\section{Definitions and preliminaries}

Throughout this paper $R$ will denote a zero-symmetric right near-ring. Unless otherwise specified, $R$ will also be assumed to contain an identity element. For any natural number $n, R^{n}$ denotes the direct sum of $n$ copies of the (not necessarily abelian) group $(R,+)$. From now on, $n$ will always denote an arbitrary but fixed natural number. We write the elements of $R^{n}$ in the form $\left\langle r_{1}, r_{2}, \ldots, r_{n}\right\rangle$ where $r_{i} \in R$ for all $i=1,2, \ldots, n$. In particular, $\overline{0}:=\langle 0,0, \ldots, 0\rangle$ where the symbol $:=$ means "is defined by". The functions $\pi_{i}: R^{n} \rightarrow R$ and $l_{i}: R \rightarrow R^{n}$ will denote the $i$ th co-ordinate projection and injection functions respectively.

Definition 1.1. The near-ring of $n \times n$-matrices over $R$, denoted by $\mathbb{M}_{n}(R)$, is defined to be the subnear-ring of $M\left(R^{n}\right)$, generated by the set of functions $\left\{f_{i j}^{r}: R^{n} \rightarrow R^{n} \mid r \in R\right.$, $1 \leqq i, j \leqq n\}$ where $f_{i j}^{r}\left\langle r_{1}, r_{2}, \ldots, r_{n}\right\rangle:=\left\langle s_{1}, s_{2}, \ldots, s_{n}\right\rangle$ with $s_{i}=r r_{j}$ and $s_{k}=0$ if $k \neq i$. The elements of $\mathbb{M}_{n}(R)$ will be referred to as $n \times n$-matrices over $R$.

It follows that $\mathbb{M}_{n}(R)$ is a zero-symmetric right near-ring with identity $I=f_{11}^{1}+f_{22}^{1}+$ $\cdots+f_{n n}^{1}$. If $R$ happens to be a ring, then $\mathbb{M}_{n}(R)$ is isomorphic to the usual full matrix ring over $R$. Sometimes, because of typographical problems, we write $f_{i j}^{r}$ as $[r ; i, j]$.

It happens frequently that we need to know a specific way in which a matrix is compiled in terms of the functions $f_{i j}^{r}$. We therefore introduce the following concept.

Definition 1.2. Let $S$ denote the free semigroup over the alphabet of symbols $\left\{f_{i j}^{r} \mid r \in R, 1 \leqq i, j \leqq n\right\} \cup\{(),+$,$\} . The set \mathbb{E}_{n}(R)$ of matrix expressions is the subset of $S$, recursively defined by the following rules:

(a) $f_{i j}^{r} \in \mathbb{E}_{n}(R)$ for all $r \in R$ and $1 \leqq i, j \leqq n$;

(b) if $X, Y \in \mathbb{E}_{n}(R)$, then $X+Y \in \mathbb{E}_{n}(R)$;

(c) if $X, Y \in \mathbb{E}_{n}(R)$, then $(X)(Y) \in \mathbb{E}_{n}(R)$;

(d) nothing else is in $\mathbb{E}_{n}(R)$.

Clearly, each element of $\mathbb{E}_{n}(R)$ represents a matrix in $\mathbb{M}_{n}(R)$. On the other hand, each matrix has infinitely many expressions representing it. For example, the expressions $X$ and $X+f_{11}^{0}$, for any $X \in \mathbb{E}_{n}(R)$, represent the same matrix. Also, when we write down an expression, we usually discard any redundant parentheses without disturbing unambiguity. For example, the expression $\left(f_{11}^{r}\right)\left(f_{11}^{s}+f_{12}^{t}\right)$ would be written (mostly) as $f_{11}^{r}\left(f_{11}^{s}+f_{12}^{t}\right)$. If $X \in \mathbb{E}_{n}(R), m(X)$ will denote the matrix in $\mathbb{M}_{n}(R)$ represented by $X$.

Definition 1.3. Let $X \in \mathbb{E}_{n}(R)$ and $U \in \mathbb{M}_{n}(R)$. The length, $l(X)$, of $X$ is defined to be the number of $f_{i j}^{r}$ in it. The weight, $w(U)$, of $U$ is defined to be the length of an expression $Y$ of minimal length such that $m(Y)=U$.

One way to relate (two-sided) ideals in $\mathbb{M}_{n}(R)$ to those in $R$, is by means of Noetherian quotients: If $A$ is an ideal of $R$ then we define $A^{*}$ to be the ideal 
$\left(A^{n}: R^{n}\right)=\left\{U \in \mathbb{M}_{n}(R) \mid U \alpha \in A^{n}\right.$ for all $\left.\alpha \in R^{n}\right\}$, where $A^{n}$ is the set $\left\{\left\langle a_{1}, a_{2}, \ldots, a_{n}\right\rangle \in R^{n} \mid\right.$ $\left.a_{i} \in A, i=1,2, \ldots, n\right\}$. As a matter of fact, if $L$ is a left ideal of $R$, then $\left(L^{n}: R^{n}\right)$ is also a two-sided ideal of $\mathbb{M}_{n}(R)$ and is equal to $A^{*}$, where $A$ is the largest two-sided ideal contained in $L$. We prove this in the following lemma.

Lemma 1.4. If $L$ is a left ideal of $R$ and $A$ is the largest two-sided ideal of $R$ contained in $L$, then $L^{*}=A^{*}$.

Proof. Since $A \subseteq L, A^{*} \subseteq L^{*}$. Now suppose $U \notin A^{*}$. Then $\pi_{i} U \alpha \notin A$ for some $i, 1 \leqq i \leqq n$, and $\alpha \in R^{n}$. Therefore, $\left(\pi_{i} U \alpha\right) r \notin L$ for some $r \in R$. But $\left(\pi_{i} U \alpha\right) r=\pi_{i} U(\alpha r)$, where $\alpha r$ means multiply each co-ordinate of $\alpha$ by $r$ on the right. (See Meyer [7, Lemma 2.1.]) Hence, $U \notin L^{*}$.

Note that there are other (non-equivalent) ways of relating ideals in $\mathbb{M}_{n}(R)$ with those of $R$, resulting in a vital difference between ring matrices and near-ring matrices, namely that there is in general not a bijection between the set of ideals of $R$ and the set of ideals of $\mathbb{M}_{n}(R)$-even if $R$ is a finite weakly distributive d.g. near-ring with identity. More details are contained in [12], [7] and [3].

Given an $R$-module $G$, one can ask the question: If $G^{n}$ is the direct sum of $n$ copies of $G$, how can we define an $M_{n}(R)$-module structure on $G^{n}$ ? We need the following definition.

Definition 1.5. Let $G$ be an $R$-module. Then $G$ is said to be locally monogenic if for any finite subset $H$ of $G$ there exists $g \in G$ such that $H \subseteq R g$.

This idea was introduced by Van der Walt [11] and he used the term connected. Clearly, if $G$ is finite, then $G$ is locally monogenic if and only if $G$ is monogenic.

Now, if $G$ is a locally monogenic $R$-module, then we define the action of $\mathrm{M}_{n}(R)$ on $G^{n}$ as follows: Let $U \in \mathbb{M}_{n}(R)$ and $\left\langle g_{1}, g_{2}, \ldots, g_{n}\right\rangle \in G^{n}$. Then, by Definition 1.5 , there are $g \in G$ and $r_{1}, r_{2}, \ldots, r_{n} \in R$ such that $g_{i}=r_{i} g, i=1,2, \ldots, n$. Let $U\left\langle g_{1}, g_{2}, \ldots, g_{n}\right\rangle:=$ $\left(U\left\langle r_{1}, r_{2}, \ldots, r_{n}\right\rangle\right) g$, where $\left\langle s_{1}, s_{2}, \ldots, s_{n}\right\rangle g:=\left\langle s_{1} g, s_{2} g, \ldots, s_{n} g\right\rangle$ for any $\left\langle s_{1}, s_{2}, \ldots, s_{n}\right\rangle \in R^{n}$. It is shown in Van der Walt [11] that this action is well-defined and it makes $G^{n}$ an $\mathrm{M}_{n}(R)$-module.

Also note that $R^{n}$ can be viewed as an $\mathbb{M}_{n}(R)$-module in a natural way, since $\mathbb{M}_{n}(R)$ is a subnear-ring of $M\left(R^{n}\right)$. If $L$ is a left ideal of $R$, then the action of $R$ on $R / L$, namely $r(s+L):=r s+L$ for all $r, s \in R$, can be used to define $(R / L)^{n}$ as an $\mathbb{M}_{n}(R)$-module as follows: Let $U \in \mathbb{M}_{n}(R)$ and $\left\langle r_{1}+L, r_{2}+L, \ldots, r_{n}+L\right\rangle \in(R / L)^{n}$ and suppose $U\left\langle r_{1}, r_{2}, \ldots, r_{n}\right\rangle=$ $\left\langle t_{1}, t_{2}, \ldots, t_{n}\right\rangle$. Then $U\left\langle r_{1}+L, r_{2}+L, \ldots, r_{n}+L\right\rangle:=\left\langle t_{1}+L, t_{2}+L, \ldots, t_{n}+L\right\rangle$. An easy induction argument on the weight of matrices in $M_{n}(R)$ shows that this action is well-defined and turns $(R / L)^{n}$ into an $\mathbb{M}_{n}(R)$-module. Furthermore, $L^{n}$ is an $\mathbb{M}_{n}(R)$-ideal of $R^{n}$ and we can therefore also consider $R^{n} / L^{n}$ as an $\mathbb{M}_{n}(R)$-module in the usual way. The following lemma states that there is virtually no difference between the $M_{n}(R)$-modules $(R / L)^{n}$ and $R^{n} / L^{n}$. 
Lemma 1.6. (Meyer [7]). If $L$ is a left ideal of $R$ then the $\mathbb{M}_{n}(R)$-modules $R^{n} / L^{n}$ and $(R / L)^{n}$ are $\mathbb{M}_{n}(R)$-isomorphic.

We now state some results which will be useful later on:

Theorem 1.7. (Van der Walt [11]). If $A$ is a two-sided ideal of $R$, then $\mathbb{M}_{n}(R / A) \cong$ $\mathbb{M}_{n}(R) / A^{*}$ as near-rings.

Lemma 1.8. (Van der Walt [11]). Let $G$ be an $R$-module and $v \in\{0,2\}$. If $R$ is $v$-primitive on $G$, then $\mathbb{M}_{n}(R)$ is v-primitive on $G^{n}$.

Lemma 1.9. (Van der Walt [11]). Let $v \in\{0,2\}$. If $A$ is a $v$-primitive ideal of $R$, then $A^{*}$ is a v-primitive ideal of $\mathbb{M}_{n}(R)$.

Lemma 1.10. (Van der Walt [11]). Suppose $\Gamma$ is a type $2 \mathbb{M}_{n}(R)$-module and let $\mathscr{A}:=\mathrm{Ann}_{\mathrm{M}_{\mathrm{s}}(R)} \Gamma$. Then there is an ideal $A$ of $R$ such that $\mathscr{A}=A^{*}$.

Lemma 1.11. (Meyer [7]). An ideal $\mathscr{A}$ of $\mathbb{M}_{n}(R)$ is 2-primitive if and only if $\mathscr{A}=A^{*}$ for some 2-primitive ideal $A$ of $R$.

Lemma 1.12. (Van der Walt [11]). If the $\mathbb{M}_{n}(R)$-module $\Gamma$ is monogenic, then $\Gamma \cong G^{n}$ as additive groups for an appropriate $R$-module $G$.

The $R$-module $G$ of Lemma 1.12 is defined as $f_{11}^{1} \Gamma=\left\{f_{11}^{1} \gamma \mid \gamma \in \Gamma\right\}$ where $r\left(f_{11}^{1} \gamma\right):=$ $f_{11}^{1}\left(f_{11}^{r} \gamma\right)$ for all $r \in R$ and $f_{11}^{1} \gamma \in f_{11}^{1} \Gamma$.

\section{Maximal left ideals}

Whilst studying 0-primitivity in matrix near-rings, it would be very handy to have some nice relationships between maximal left ideals of $R$ and those of $M_{n}(R)$. Stone [10] characterises all maximal left ideals in matrix rings as follows:

Theorem 2.1. (Stone [10]). If $L$ is a maximal left ideal of a ring $R$ and $\alpha \in R^{n} \backslash L^{n}$, then $\left(L^{n}: \alpha\right):=\left\{U \in \mathbb{M}_{n}(R) \mid U \alpha \in L^{n}\right\}$ is a maximal left ideal of $\mathbb{M}_{n}(R)$. Moreover, every maximal left ideal of $\mathbb{M}_{n}(R)$ is of this form.

Unfortunately, in the near-ring case the situation is not the same. We will show that under certain conditions, $\left(L^{n}: \alpha\right)$ is indeed a maximal left ideal of $\mathbb{M}_{n}(R)$, where $R$ is a zero-symmetric near-ring with identity (Theorem 2.4), but not under the general conditions of Theorem 2.1 (Example 2.5). Also, we will prove that for some "wellbehaved" near-rings $R$, the maximal left ideals of $\mathbb{M}_{n}(R)$ are indeed of the form $\left(L^{n}: \alpha\right)$ as described in Theorem 2.1 (Theorem 2.11). Before we can prove these theorems, we need the following lemmas. 
Lemma 2.2. Let $A=\left\{s_{1}, s_{2}, \ldots, s_{n}\right\}$ be a finite subset of $R$ and let $S$ be the $R$-subgroup of $R$ generated by $A$. Furthermore, let $T$ be the subset of $R$ recursively defined by the following rules:

(a) $s_{i} \in T$ for all $i=1,2, \ldots, n$;

(b) if $t_{1}, t_{2} \in T$, then $t_{1}-t_{2} \in T$;

(c) if $t \in T$ and $r \in R$, then $r t \in T$;

(d) nothing else is in $T$.

Then $S=T$.

Proof. First of all, that $T$ is an $R$-subgroup of $R$, follows directly from (b) and (c). Since $A \subseteq T$ (by (a)), we must have $S \subseteq T$.

Before showing that $T \subseteq S$, let us introduce some more terminology. Each $t \in T$ is always constructed (in many ways) by a finite number of applications of the rules (a)(c), starting always with rule (a). A unique number $c_{A}(t)$ which is in effect the minimum number of applications of the rules (a)-(c) needed to construct $t$, will be assigned to $t$ in the following way:

We call a sequence $t_{1}, t_{2}, \ldots, t_{m}$ of elements of $T$ a generating sequence of length $m$ for $t$ with respect to $A$ if $t_{1} \in A, t_{m}=t$ and for each $k=2,3, \ldots, m$, one of the following applies:

(i) $t_{k} \in A$;

(ii) $t_{k}=t_{i}-t_{j}, 1 \leqq i, j<k$;

(iii) $t_{k}=r t_{i}, 1 \leqq i<k$ and $r \in R$.

The complexity of $t$ with respect to $A$, denoted by $c_{A}(t)$, is the length of a generating sequence of minimal length for $t$ with respect to $A$. Note that $c_{A}(t)=1$ if and only if $t \in A$. We can now finish the proof of Lemma 2.2 .

Let $t \in T$. We will show that $t \in S$ by using induction on $c_{A}(t)$. If $c_{A}(t)=1$, then $t \in A \subseteq S$. Suppose $c_{A}(t)=m>1$ and that all $t^{\prime} \in T$ with $c_{A}\left(t^{\prime}\right)<m$ are contained in $S$. We have two possibilities:

1. $t=t_{1}-t_{2}$ where $t_{1}, t_{2} \in T$ and $c_{A}\left(t_{1}\right), c_{A}\left(t_{2}\right)<m$. Since $t_{1}, t_{2} \in S$, we must have $t=t_{1}-t_{2} \in S$.

2. $t=r t_{1}$, where $t_{1} \in T, r \in R$ and $c_{A}\left(t_{1}\right)<m$. Since $t_{1} \in S$, we have $t=r t_{1} \in S$.

By induction all elements of $T$ are contained in $S$ and the proof of the lemma is accomplished.

Lemma 2.3. Suppose $S$ is an $R$-subgroup of $R$ generated (as an $R$-subgroup) by the elements $s_{1}, s_{2}, \ldots, s_{n}$ in $R$. Let $\alpha:=\left\langle s_{1}, s_{2}, \ldots, s_{n}\right\rangle \in R^{n}$. Then

$$
\mathbb{M}_{n}(R) \alpha=S^{n}
$$


where $\mathbb{M}_{n}(R) \alpha:=\left\{U \alpha \mid U \in \mathbb{M}_{n}(R)\right\}$ and $S^{n}:=\left\{\left\langle x_{1}, x_{2}, \ldots, x_{n}\right\rangle \in R^{n} \mid x_{i} \in S, i=1,2, \ldots, n\right\}$.

Proof. To show that $\mathbb{M}_{n}(R) \alpha \subseteq S^{n}$, we use induction on the weight of matrices in $\mathbb{M}_{n}(R)$. Let $U \in \mathbb{M}_{n}(R)$ and suppose $w(U)=1$, i.e. $U=f_{i j}^{r}$ for some $r \in R$ and $1 \leqq i, j \leqq n$. Then $U \beta=l_{i}\left(r \pi_{j} \beta\right) \in S^{n}$, for all $\beta \in S^{n}$. In particular $U \alpha \in S^{n}$. Now suppose $w(U)=m>1$ and $V \beta \in S^{n}$ for all $\beta \in S^{n}$ and for all $V \in \mathbb{M}_{n}(R)$ with $w(V)<m$. There are two cases to consider:

1. $U=V_{1}+V_{2}$ with $V_{1}, V_{2} \in \mathbb{M}_{n}(R)$ and $w\left(V_{1}\right), w\left(V_{2}\right)<m$. It follows that $U \beta=V_{1} \beta+$ $V_{2} \beta \in S^{n}+S^{n} \subseteq S^{n}$.

2. $U=V_{1} V_{2}$ with $V_{1}, V_{2} \in \mathbb{M}_{n}(R)$ and $w\left(V_{1}\right), w\left(V_{2}\right)<m$. In this case $U \beta=\left(V_{1} V_{2}\right) \beta=$ $V_{1}\left(V_{2} \beta\right)=V_{1} \gamma$ for some $\gamma \in S^{n}$ so that $V_{1} \gamma \in S^{n}$.

In both cases it follows that $U \alpha \in S^{n}$, since $\alpha \in S^{n}$. From induction it follows now that $\mathbb{M}_{n}(R) \alpha \subseteq S^{n}$.

In order to prove that $S^{n} \subseteq \mathbb{M}_{n}(R) \alpha$, we will show that $t_{1} \pi_{1}\left(S^{n}\right)=\langle S,\{0\},\{0\}, \ldots,\{0\}\rangle \subseteq$ $\mathbb{M}_{n}(R) \alpha$. The same method can then be used to show that $t_{i} \pi_{i}\left(S^{n}\right) \subseteq \mathbb{M}_{n}(R) \alpha$ for all $i=1,2, \ldots, n$. Since $\mathbb{M}_{n}(R) \alpha$ is an $\mathbb{M}_{n}(R)$-subgroup of the $\mathbb{M}_{n}(R)$-module $R^{n}$, it follows that $\sum_{i=1}^{n} l_{i} \pi_{i}\left(S^{n}\right)=S^{n} \subseteq \mathbb{M}_{n}(R) \alpha$.

Since $S$ is the $R$-subgroup of $R$ generated by $A=\left\{s_{1}, s_{2}, \ldots, s_{n}\right\}$, we can apply Lemma 2.2 and so each element of $S$ has a complexity with respect to $A$. Now let $s \in S$ such that $c_{A}(s)=1$. Then $s \in A$, i.e. $s=s_{j}$ for some $j, 1 \leqq j \leqq n$. But then $l_{1}(s)=\langle s, 0,0, \ldots, 0\rangle=$ $f_{1 j}^{1} \alpha \in \mathbb{M}_{n}(R) \alpha$. Now suppose $s \in S$ with $c_{A}(s)=m>1$ and that $l_{1}(t) \in \mathbb{M}_{n}(R) \alpha$ for all $t \in S$ with $c_{A}(t)<m$. Consider the following possibilities:

1. $s=t_{1}-t_{2}$ with $t_{1}, t_{2} \in S$ and $c_{A}\left(t_{1}\right), \quad c_{A}\left(t_{2}\right)<m$. But then $l_{1}(s)=l_{1}\left(t_{1}\right)-$ $l_{1}\left(t_{2}\right) \in \mathbb{M}_{n}(R) \alpha-\mathbb{M}_{n}(R) \alpha \subseteq \mathbb{M}_{n}(R) \alpha$.

2. $s=r t$ where $r \in R, t \in S$ and $c_{A}(t)<m$. In this case $l_{1}(s)=f_{11}^{r} l_{1}(t) \in f_{11}^{r} \mathbb{M}_{n}(R) \alpha \subseteq$ $\mathbb{M}_{n}(R) \alpha$.

The principle of induction assures us that $l_{1}(S)=l_{1} \pi_{1}\left(S^{n}\right) \subseteq \mathbb{M}_{n}(R) \alpha$ and by the arguments above, our proof is complete.

Theorem 2.4. Suppose $L$ is a maximal left ideal of $R$ and $\alpha=\left\langle s_{1}, s_{2}, \ldots, s_{n}\right\rangle \in R^{n} \backslash L^{n}$ is such that the set $\left\{s_{1}, s_{2}, \ldots, s_{n}\right\}$ generates $R$ as an $R$-subgroup of $R$ (for example, if at least one $\left.s_{i}=1\right)$. Then $\left(L^{n}: \alpha\right)$ is a maximal left ideal of $\mathbb{M}_{n}(R)$, where $\left(L^{n}: \alpha\right):=$ $\left\{U \in \mathbb{M}_{n}(R) \mid U \alpha \in L^{n}\right\}$.

Proof. Consider the $\mathbb{M}_{n}(R)$-homomorphisms $\phi: \mathbb{M}_{n}(R) \rightarrow R^{n}$ and $\psi: R^{n} \rightarrow R^{n} / L^{n} \cong$ $(R / L)^{n}$, where $\phi(U):=U \alpha$ for all $U \in \mathbb{M}_{n}(R)$ and $\psi$ is the canonical $\mathbb{M}_{n}(R)$-epimorphism. The isomorphism follows from Lemma 1.6. Furthermore, $\mathbb{M}_{n}(R) \alpha=R^{n}$ as follows from Lemma 2.3, which means that $\phi$ is an epimorphism. But then $\psi \circ \phi: \mathbb{M}_{n}(R) \rightarrow R^{n} / L^{n}$ is an epimorphism. We deduce that $\mathbb{M}_{n}(R) /\left(L^{n}: \alpha\right)=\mathbb{M}_{n}(R) / \operatorname{Ker}(\psi \circ \phi) \cong \operatorname{Im}(\psi \circ \phi)=R^{n} / L^{n}$. But since $R / L$ is simple as $R$-module, $(R / L)^{n}$ is simple as an $\mathbb{M}_{n}(R)$-module. (See Meyer [7, 
Corollary 2.10.]) This means that $\mathbb{M}_{n}(R) /\left(L^{n}: \alpha\right) \cong R^{n} / L^{n} \cong(R / L)^{n}$ is simple as $\mathbb{M}_{n}(R)$-module and we deduce that $\left(L^{n}: \alpha\right)$ is maximal in $\mathbb{M}_{n}(R)$.

We will now provide an example to show that when $\alpha \in R^{n} \backslash L^{n}$, but the co-ordinates of $\alpha$ do not generate $R$ as $R$-subgroup of $R$, then Theorem 2.4 is in general not valid.

Example 2.5. Let $G:=\{0,1,2, \ldots, 7\}$ denote the cyclic group of order 8. The nontrivial proper subgroups of $G$ are denoted by $H_{1}:=\{0,2,4,6\}$ and $H_{2}:=\{0,4\}$. Define $R$ as follows:

$$
\begin{aligned}
R:= & \left\{f \in M_{0}(G) \mid f\left(H_{i}\right) \subseteq H_{i}, i=1,2, \text { and if } x, y \in H_{1} \text { with } x-y \in H_{2},\right. \\
& \text { then } \left.f(x)-f(y) \in H_{2}\right\}
\end{aligned}
$$

It is routine verification to check that $R$ is a zero-symmetric, abelian near-ring with identity. Moreover, $R$ is finite with $|R|=2^{16}=65536$.

Now consider the following subsets of $R$ :

$$
\begin{aligned}
& M:=\left\{f \in R \mid f(1) \in H_{1}\right\}, \\
& K:=\left\{f \in R \mid f(1) \in H_{2}\right\}, \\
& L:=\{f \in R \mid f(1)=0\}=\operatorname{Ann}_{R}(1) .
\end{aligned}
$$

Obviously, $\{0\} \subset L \subset K \subset M \subset R$, where " $\subset$ " means proper inclusion. We also observe the following facts:

I. $L$ is a maximal left ideal of $R$.

Proof. Being the annihilator of an element in $G, L$ is certainly a left ideal of $R$. Since $R 1=G$, we have that $R / \operatorname{Ann}_{R}(1)=R / L \cong G$ as $R$-modules. The only possible non-trivial proper $R$-ideals of $G$ are $H_{1}$ and $H_{2}$. But $r(2+1)-r(1)=$ $r(3)-r(1)=1$ if $r(3)=1$ and $r(x)=0$ if $x \neq 3$. Since $2 \in H_{1}$ and $1 \notin H_{1}, H_{1}$ is not an $R$-ideal of $G$. In a similar way it follows that $H_{2}$ neither is an $R$-ideal of $G$, implying that $G$ is a simple $R$-module. But then $R / L$ is a simple $R$-module and so $L$ is a maximal left ideal of $R$.

II. Both $K$ and $M$ are $R$-subgroups of $R$ (and not $R$-ideals).

Proof. Straightforward.

1II. $K$ is an $R$-ideal of $M$.

Proof. Since $(K,+)$ is a normal subgroup of $(R,+)$, it is a normal subgroup of $(M,+)$ as well. Let $k \in K, m \in M$ and $r \in R$. Then

$$
\begin{aligned}
{[r(k+m)-r m](1)=} & r\left(h_{2}+h_{1}\right)-r\left(h_{1}\right) \text { where } h_{i} \in H_{i}, i=1,2 \\
& \in H_{2}, \text { since } h_{1}, h_{1}+h_{2} \in H_{1} \text { and }\left(h_{1}+h_{2}\right)-h_{1} \in H_{2} .
\end{aligned}
$$


IV. We have the following proper inclusions of $R$-modules:

$$
L / L \subset K / L \subset M / L \text {. }
$$

Proof. This is merely a matter of equivalence class arithmetic.

V. The $R$-module $M / L$ is not simple.

Proof. From III and IV it follows readily that $K / L$ is a non-trivial proper $R$-ideal of $M / L$.

VI. The $R$-subgroup $M$ of $R$ is generated (as an $R$-subgroup) by the two elements $m_{1}$ and $m_{2}$, where

$$
m_{1}(x):=\left\{\begin{array}{c}
2 \text { if } x=1 \\
0 \text { if } x=2 \\
4 \text { if } x=6 \\
x \text { otherwise }
\end{array} \quad m_{2}(x):=\left\{\begin{array}{c}
2 \text { if } x=1 \\
0 \text { if } x=4 \\
x \text { otherwise. }
\end{array}\right.\right.
$$

Proof. Since $m_{1}, m_{2} \in M$, the $R$-subgroup generated by $m_{1}$ and $m_{2}$ is certainly contained in $M$. Conversely, if $m \in M$, choose $r_{1}, r_{2} \in R$ as follows:

$$
r_{1}(x):=\left\{\begin{array}{cc}
m(1)-m(2) & \text { if } x=2 \\
m(1)+m(2) & \text { if } x=6 \\
m(x) & \text { otherwise }
\end{array} \quad r_{2}(x):=\left\{\begin{array}{cc}
m(2) & \text { if } x=2 \\
m(6)-m(4) & \text { if } x=6 \\
0 & \text { otherwise }
\end{array}\right.\right.
$$

Then $r_{1} m_{1}+r_{2} m_{2}=m$, as can be easily verified and so $M$ is contained in the $R$-subgroup generated by $m_{1}$ and $m_{2}$.

VII. For any $n \geqq 2$ we have that $\mathbb{M}_{n}(R) \alpha=M^{n}$, where $\alpha:=\left\langle m_{1}, m_{2}, 0,0, \ldots, 0\right\rangle \in R^{n}$ with $m_{1}$ and $m_{2}$ as in $V I$.

Proof. This result follows directly from VI and Lemma 2.3.

VIII. For the $\alpha$ of VII it follows that $\alpha \in R^{n} \backslash L^{n}$ and $\left(L^{n}: \alpha\right)$ is not a maximal left ideal of $\mathbb{M}_{n}(R)$.

Proof. Consider the mappings $\phi: \mathbb{M}_{n}(R) \rightarrow R^{n}$ and $\psi: R^{n} \rightarrow R^{n} / L^{n} \cong(R / L)^{n}$ of $\mathbb{M}_{n}(R)$-modules as in the proof of Theorem 2.4. It follows that

$$
\begin{aligned}
\operatorname{Im}(\psi \circ \phi) & =\left\{U \alpha+L^{n} \mid U \in \mathbb{M}_{n}(R)\right\} \\
& =M^{n} / L^{n} \text { by VII. }
\end{aligned}
$$

Furthermore, $\mathbb{M}_{n}(R) /\left(L^{n}: \alpha\right)=\mathbb{M}_{n}(R) / \operatorname{Ker}(\psi \circ \phi) \cong M^{n} / L^{n}$. But $M / L$ is not simple as an $R$-module (from V) and so $(M / L)^{n} \cong M^{n} / L^{n}$ is not simple as an $\mathbb{M}_{n}(R)$-module which implies that $\left(L^{n}: \alpha\right)$ is not maximal in $\mathbb{M}_{n}(R)$.

It must be emphasised that although $K$ is not a left ideal of $R,\left(K^{n}: \alpha\right)$ is indeed a maximal left ideal of $\mathbb{M}_{n}(R)$, properly containing $\left(L^{n}: \alpha\right)$. It can be shown that $\left(K^{n}: \alpha\right)$ is of the form $\left(T^{n}: \beta\right)$ where $T$ is a maximal left ideal of $R$ and $\beta \in R^{n} \backslash T^{n}$ : Take $T$ as $\left\{f \in R \mid f(2), f(6) \in H_{2}\right\}$, and $\beta=\langle 1,1,0,0, \ldots, 0\rangle \in R^{n}$.

If $\Gamma$ is a faithful type $0 \mathbb{M}_{n}(R)$-module, then $\Gamma$ is $\mathbb{M}_{n}(R)$-isomorphic to $\mathbb{M}_{n}(R) / \mathscr{L}$ for some maximal left ideal $\mathscr{L}$ of $M_{n}(R)$. It follows from faithfulness that the largest 
two-sided ideal in $\mathscr{L}$ is $\{0\}$ and hence, if $\mathscr{L}=\left(L^{n}: \alpha\right)$ for some maximal left ideal $L$ of $R$ and $\alpha \in R^{n} \backslash L^{n}$, then $L^{*}=\{0\}$, because $L^{*}=\left(L^{n}: R^{n}\right) \subseteq\left(L^{n}: \alpha\right)=\mathscr{L}$ and $L^{*}$ is two-sided. Consequently, if we can find an $R$ with $\mathbb{M}_{n}(R) 0$-primitive and such that no maximal left ideal $L$ of $R$ has the property $L^{*}=\{0\}$, then at least one maximal left ideal of $\mathbb{M}_{n}(R)$ cannot be written in the form $\left(L^{n}: \alpha\right)$ where $\alpha \in R^{n} \backslash L^{n}$. It is not known whether such an $R$ exists. In Theorem 2.11 , however, it will be shown that when $R$ is a weakly distributive d.g. near-ring, then every maximal left ideal of $\mathbb{M}_{n}(R)$ can be expressed in this form.

Recall that a d.g. near-ring $R$ is weakly distributive if its distributor series $\left\{D^{i}(R)\right\}$ terminates in $\{0\}$, where

$$
\begin{gathered}
D^{0}(R):=R, \text { and } \\
D^{i+1}(R):=G p\left\langle\left\{x(a+b)-x b-x a \mid x \in R, a, b \in D^{i}(R)\right\}\right\rangle^{R} \text { if } i \geqq 0 .
\end{gathered}
$$

Here $G p\langle X\rangle^{R}$ denotes the normal subgroup of $(R,+)$ generated by $X \subseteq R$. The interested reader should consult Meldrum [4] for a comprehensive study on this subject. We also quote the following lemmas from [4]:

Lemma 2.6. (Meldrum [4, Theorem 9.45]). Let $R$ be a d.g. near-ring with $R^{2}=R$. Then $D^{n}(R)=\delta_{n}(R)$ for all $n \geqq 0$ where $\delta_{n}(R)$ denotes the nth term of the derived series of the group $(R,+)$.

Lemma 2.7. (Meldrum [4, Corollary 9.46]). If $R$ is a d.g. near-ring with $R^{2}=R$, then $R$ is weakly distributive if and only if $(R,+)$ is soluble.

Lemma 2.8. (Meldrum [4, Corollary 9.34]). If $R$ is a d.g. near-ring then $\delta_{i}(R)$ is an ideal of $R$ for all $i \geqq 0$.

Lemma 2.9. (Meldrum [4, Corollary 9.49]). If $R$ is a d.g. near-ring with $(R,+)$ soluble, then $\delta_{1}(R)$ is multiplicatively nilpotent.

It was shown in Abbasi, Meldrum and Meyer [2] that if $R$ is a weakly distributive d.g. near-ring, then so is $\mathbb{M}_{n}(R)$. By Lemmas $2.7,2.8$ and 2.9 it follows that $\delta_{1}\left(\mathbb{M}_{n}(R)\right)$ is a multiplicatively nilpotent ideal of $\mathbb{M}_{n}(R)$. Consequently, $\delta_{1}\left(\mathbb{M}_{n}(R)\right)$ is contained in $\mathscr{T}_{1 / 2}\left(\mathbb{M}_{n}(R)\right)$ from which it follows that $\delta_{1}\left(\mathbb{M}_{n}(R)\right) \subseteq \mathscr{L}$ for any maximal left ideal $\mathscr{L}$ of $\mathbb{M}_{n}(R)$, since $\mathscr{T}_{1 / 2}\left(\mathbb{M}_{n}(R)\right)=\cap\left\{\mathscr{L} \mid \mathscr{L}\right.$ is a maximal left ideal of $\left.\mathbb{M}_{n}(R)\right\}$. This leads us to the following lemma:

Lemma 2.10. Suppose $R$ is a weakly distributive d.g. near-ring and let $\mathscr{L}$ be a maximal left ideal of $\mathbb{M}_{n}(R)$. Then there exists an $\alpha \in R^{n}$ such that the set of co-ordinates of $\alpha$ generates $R$ as an $R$-subgroup and such that $(\mathscr{L} \alpha: \alpha):=\left\{U \in \mathbb{M}_{n}(R) \mid U \alpha \in \mathscr{L} \alpha\right\} \subset \mathbb{M}_{n}(R)$, where $\mathscr{L} \alpha:=\{L \alpha \mid L \in \mathscr{L}\}$.

Proof. Since $\mathbb{M}_{n}(R)$ is d.g., each matrix can be represented by an expression involving only $f_{i j}^{r}$ and plus-signs (Abbasi [1, Theorem 4.1]). In fact, since $\mathbb{M}_{n}(R)$ is also weakly distributive, any $U \in \mathbb{M}_{n}(R)$ can be expressed as 


$$
\begin{aligned}
U= & f_{11}^{r_{11}}+f_{12}^{r_{12}}+\cdots+f_{1 n}^{r_{1 n}} \\
& +f_{21}^{r_{21}}+f_{22}^{r_{22}}+\cdots+f_{2 n}^{r_{2 n}} \\
& \vdots \quad \vdots \quad \ddots \quad \vdots \\
& +f_{n 1}^{r_{n 1}}+f_{n 2}^{r_{22}}+\cdots+f_{n n}^{r_{n n}}+U^{\prime}, \text { where } U^{\prime} \in \delta_{1}\left(\mathbb{M}_{n}(R)\right) \subseteq \mathscr{L} .
\end{aligned}
$$

Now suppose the lemma is not true. Then $(\mathscr{L} \alpha: \alpha)=\mathbb{M}_{n}(R)$ for all $\alpha \in R^{n}$ of which the co-ordinates form a generating set for $R$ as $R$-subgroup; in particular, for all $\alpha$ with $\pi_{i} \alpha=1$ for some $i, 1 \leqq i \leqq n$. Consequently, $\mathscr{L} \alpha=R^{n}$ for all such $\alpha$. To simplify matters, we shall stick to the case $n=2$. A similar (but much more clumsy) procedure applies for the case $n>2$.

For every $y \in R$ there is a matrix $U_{y} \in \mathscr{L}$ such that $U_{y}\langle 1, y\rangle=\langle 1,0\rangle$. Since $f_{11}^{1} U_{y} \in \mathscr{L}$ and $f_{11}^{1} U_{y}\langle 1, y\rangle=\langle 1,0\rangle$, we shall only consider first row matrices in $\mathscr{L}$, i.e. matrices of the form $f_{11}^{1} L, L \in \mathscr{L}$. Similarly, for every $x \in R$, there is a (first row) matrix $V_{x} \in \mathscr{L}$ such that $V_{x}\langle x, 1\rangle=\langle 1,0\rangle$. Now suppose

$$
U_{y}=\left[r_{1} ; 1,1\right]+\left[s_{1} ; 1,2\right]+\left[r_{2} ; 1,1\right]+\left[s_{2} ; 1,2\right]+\cdots+\left[r_{m} ; 1,1\right]+\left[s_{m} ; 1,2\right] .
$$

Then

$$
U_{y}=\left[r_{1}+r_{2}+\cdots+r_{m} ; 1,1\right]+\left[s_{1}+s_{2}+\cdots+s_{m} ; 1,2\right]+U_{y}^{\prime} \text { for some } U_{y}^{\prime} \in \mathscr{L} .
$$

Let $a(y):=r_{1}+r_{2}+\cdots+r_{m}$ and $b(y):=s_{1}+s_{2}+\cdots+s_{m}$. Then, since $U_{y}\langle 1, y\rangle=\langle 1,0\rangle$, it follows that $a(y)+b(y) y+d(y)=1$ for some $d(y) \in \delta_{1}(R)$. Consequently, for any $y \in R$, there aie $b(y) \in R$ and $d(y) \in \delta_{1}(R)$ such that

$$
[1-d(y)-b(y) y ; 1,1]+[b(y) ; 1,2] \in \mathscr{L} .
$$

But $[-d(y) ; 1,1] \in \mathscr{L}$ (Abbasi [1, Corollary 4.18]) and thus we have that

$$
[1-b(y) y ; 1,1]+[b(y) ; 1,2] \in \mathscr{L} .
$$

By a similar argument, for any $x \in R$, there is an $a(x) \in R$ such that

$$
[a(x) ; 1,1]+[1-a(x) x ; 1,2] \in \mathscr{L} .
$$

Since $\mathscr{L}$ is a left ideal we deduce that for any $x, y, z, w \in R,[z(1-b(y) y) ; 1,1]+$ $[z b(y) ; 1,2] \in \mathscr{L}$ and $[w a(x) ; 1,1]+[w(1-a(x) x) ; 1,2] \in \mathscr{L}$, and so

$$
[z(1-b(y) y)+w a(x) ; 1,1]+[z b(y)+w(1-a(x) x) ; 1,2] \in \mathscr{L} .
$$

Let $y=0, x=-b(0), w=-b(0)$ and $z=1+b(0) a(-b(0))$. Then we have (with $b(0)$ written as $b$ and using the fact that $x(-y)-x y \in \delta_{1}(R)$ for all $\left.x, y \in R\right)$

$$
[1 ; 1,1]+[b+b a(-b) b+(-b)(1+a(-b) b) ; 1,2] \in \mathscr{L}
$$


and since the expression in $a$ and $b$ is an element of $D^{1}(R)=\delta_{1}(R)$, we conclude that

$$
f_{11}^{1} \in \mathscr{L}
$$

It follows mutatis mutandis that $f_{22}^{1} \in \mathscr{L}$ and therefore $f_{11}^{1}+f_{22}^{1}$, the identity matrix, is an element of $\mathscr{L}$, which is a contradiction.

Theorem 2.11. If $R$ is a weakly distributive d.g. near-ring and $\mathscr{L}$ is a maximal left ideal of $\mathbb{M}_{n}(R)$, then there exists a maximal left ideal $L$ of $R$ such that $\mathscr{L}=\left(L^{n}: \alpha\right)$ for some $\alpha \in R^{n} \backslash L^{n}$.

Proof. From the previous lemma it follows that there is an $\alpha \in R^{n}$ (of which the co-ordinates generate $R$ as an $R$-subgroup and can therefore not be in $L^{n}$ for any proper left ideal $L$ of $R)$ such that $(\mathscr{L} \alpha: \alpha) \subset \mathbb{M}_{n}(R)$. But since $\mathscr{L} \subseteq(\mathscr{L} \alpha: \alpha)$ and $\mathscr{L}$ is maximal, we must have $\mathscr{L}=(\mathscr{L} \alpha: \alpha)$. Also, $\mathscr{L} \alpha$ is an $\mathbb{M}_{n}(R)$-ideal of the $\mathbb{M}_{n}(R)$-module $R^{n}$ and is thus of the form $K^{n}$ for some left ideal $K$ of $R$ (Van der Walt [11, Lemma 3.7]). But $K$ is contained in a maximal left ideal $L$ which means that $\mathscr{L}=\left(K^{n}: \alpha\right) \subseteq\left(L^{n}: \alpha\right) \subset \mathbb{M}_{n}(R)$ so that $\mathscr{L}=\left(L^{n}: \alpha\right)$.

Corollary 2.12. If the d.g. near-ring $R$ is weakly distributive, then

$$
\left(\mathscr{T}_{1 / 2}(R)\right)^{*}=\mathscr{T}_{0}\left(\mathbb{M}_{n}(R)\right)=\left(\mathscr{T}_{0}(R)\right)^{*}
$$

Proof.

$$
\begin{aligned}
\mathscr{T}_{1 / 2}\left(\mathbb{M}_{n}(R)\right)= & \cap\left\{\mathscr{L} \mid \mathscr{L} \text { is a maximal left ideal of } \mathbb{M}_{n}(R)\right\} \\
= & \cap\left\{\left(L^{n}: \alpha_{L}\right) \mid L\right. \text { is an element of a subset of the set of all maximal } \\
& \left.\quad \text { eft ideals of } R \text { and } \alpha_{L} \in R^{n} \backslash L^{n}\right\}, \text { by Theorem } 2.11 \\
\supseteq & \cap\left\{\left(L^{n}: \alpha\right) \mid L \text { is a maximal left ideal of } R \text { and } \alpha \in R^{n} \backslash L^{n}\right\} \\
\supseteq & \cap\left\{\left(L^{n}: R^{n}\right) \mid L \text { is a maximal left ideal of } R\right\} \\
= & \left((\cap\{L \mid L \text { is a maximal left ideal of } R\})^{n}: R^{n}\right) \text { by Pilz }[9,1.44] \\
= & \left(\left(\mathscr{T}_{1 / 2}(R)\right)^{n}: R^{n}\right) \\
= & \left(\mathscr{T}_{1 / 2}(R)\right)^{*} .
\end{aligned}
$$

Since $\left(\mathscr{T}_{1 / 2}(R)\right)^{*}$ is two-sided, $\left(\mathscr{T}_{1 / 2}(R)\right)^{*} \subseteq \mathscr{T}_{0}\left(\mathbb{M}_{n}(R)\right)$. Furthermore, $\mathscr{T}_{0}\left(\mathbb{M}_{n}(R)\right) \subseteq\left(\mathscr{T}_{0}(R)\right)^{*}$, from Meyer [7, Theorem 2.34(a)], and since $\left(\mathscr{T}_{0}(R)\right)^{*}=\left(\mathscr{T}_{1 / 2}(R)\right)^{*}$ (by Lemma 1.4), the result follows. 


\section{0-Primitivity}

In this section we will concentrate on those $R$-modules embeddable into ${ }_{R} R$. We shall see that when $R$ has $D C C R$, i.e. $R$ has the descending chain condition on $R$-subgroups, then much can be said about simple faithful $R$-subgroups of $R$. If $R$ is finite we can even go further and prove a strong relationship between $R$ and $\mathbb{M}_{n}(R)$, as far as 0-primitivity is concerned. Of course, the next step would be to study this relationship in arbitrary zero-symmetric near-rings.

Lemma 3.1. Suppose $K$ is an $R$-subgroup of $R$. Then

(a) The $R$-module $K$ is faithful if and only if the $\mathbb{M}_{n}(R)$-module $K^{n}$ is faithful.

(b) The $R$-module $K$ is simple if and only if the $\mathbb{M}_{n}(R)$-module $K^{n}$ is simple.

Proof. (a) Suppose $M_{n}(R) K^{n}$ is faithful. Let $0 \neq r \in R$. Then $f_{11}^{r}$ is non-zero in $M_{n}(R)$ which means that there is an $\alpha \in K^{n}$ such that $f_{11}^{r} \alpha \neq \overline{0}$. This implies that $\pi_{1} \alpha \in K$ and $r\left(\pi_{1} \alpha\right) \neq 0$. Consequently, ${ }_{R} K$ is faithful.

On the other hand, let ${ }_{R} K$ be faithful. Suppose $U \in \mathbb{M}_{n}(R)$ is non-zero. Then $U\left\langle r_{1}, r_{2}, \ldots, r_{n}\right\rangle=\left\langle t_{1}, t_{2}, \ldots, t_{n}\right\rangle$ with $r_{i}, t_{i} \in R$ and at least one $t_{i}$, say $t_{1}$, is non-zero. Since ${ }_{R} K$ is faithful, there is a $k \in K$ such that $t_{1} k \neq 0$. But then $U\left\langle r_{1} k, r_{2} k, \ldots, r_{n} k\right\rangle=$ $\left\langle t_{1} k, t_{2} k, \ldots, t_{n} k\right\rangle \neq \overline{0}$, while $\left\langle r_{1} k, r_{2} k, \ldots, r_{n} k\right\rangle \in K^{n}$. In other words, ${ }_{\mathbf{N}_{2}(R)} K^{n}$ is faithful.

(b) Suppose ${ }_{R} K$ is not simple. Then there exists an $R$-ideal $H$ of $K$ such that $\{0\} \subset H \subset K$ and so $\left(H^{n},+\right)$ is a proper non-trivial normal subgroup of $\left(K^{n},+\right)$. Moreover, $H^{n}$ is an $\mathbb{M}_{n}(R)$-ideal of $K^{n}$, as follows: Let $\alpha \in H^{n}, \beta \in K^{n}$ and $f_{i j}^{r} \in \mathbb{M}_{n}(R)$. Then $f_{i j}^{r}(\alpha+\beta)-f_{i j}^{r} \beta=\gamma$, where $\pi_{i} \gamma \in H$ and $\pi_{k} \gamma=0$ if $k \neq i$. So $\gamma \in H^{n}$. Now let $w(U)=m>1$, and suppose $V(\alpha+\beta)-V \beta \in H^{n}$ for all $\alpha \in H^{n}, \beta \in K^{n}$ and matrices $V$ with $w(V)<m$. There are two cases to consider:

1. $U=V_{1}+V_{2}$, with $w\left(V_{1}\right), w\left(V_{2}\right)<m$. But then $U(\alpha+\beta)-U \beta=\left(V_{1}+V_{2}\right)(\alpha+\beta)-$ $\left(V_{1}+V_{2}\right) \beta=V_{1}(\alpha+\beta)+V_{2}(\alpha+\beta)-V_{2} \beta-V_{1} \beta=V_{1}(\alpha+\beta)+\gamma-V_{1} \beta=V_{1}(\alpha+\beta)-$ $V_{1} \beta+\gamma^{\prime} \in H^{n}$, for some $\gamma, \gamma^{\prime} \in H^{n}$.

2. $U=V_{1} V_{2}$, with $w\left(V_{1}\right), w\left(V_{2}\right)<m$. In this case, $U(\alpha+\beta)-U \beta=V_{1} V_{2}(\alpha+\beta)-$ $V_{1} V_{2} \beta=V_{1}\left[V_{2}(\alpha+\beta)-V_{2} \beta+V_{2} \beta\right]-V_{1} V_{2} \beta \in H^{n}$, since $V_{2}(\alpha+\beta)-V_{2} \beta \in H^{n}$.

From induction it follows that ${ }_{M_{n}(R)} K^{n}$ is not simple.

Conversely, suppose $M_{d}(R) K^{n}$ is not simple. Then there is a non-trivial $\mathbb{M}_{n}(R)$-ideal $\mathscr{H} \subset K^{n}$. But $\mathscr{H}$ is of the form $H^{n}$ for some $R$-ideal $H$ of $K$, where $\{0\} \subset H \subset K$ (take $H=\left\{\pi_{1} \alpha \mid \alpha \in \mathscr{H}\right\}$.) As a consequence, ${ }_{R} K$ is not simple.

Theorem 3.2. Suppose $R$ has DCCR and does not necessarily contain an identity. Let $K$ be a non-zero $R$-subgroup of $R$. If the $R$-module $K$ is simple and faithful, then it is monogenic.

Proof. Since $K$ is faithful, $K \nsubseteq \mathrm{Ann}_{R}\left(k_{1}\right)$ for some $k_{1} \in K$. Moreover, because $K \cap \operatorname{Ann}_{R}\left(k_{1}\right)$ is an $R$-ideal of $K$, we must have $K \cap \operatorname{Ann}_{R}\left(k_{1}\right)=\{0\}$. Now consider the map $\phi: K \rightarrow K$ where $\phi(k):=k k_{1}$ for all $k \in K$. This map is injective, for if $k k_{1}=k^{\prime} k_{1}$ where $k \neq k^{\prime}$, then $0 \neq k-k^{\prime} \in K \cap A n_{R}\left(k_{1}\right)=\{0\}$, a contradiction. That $\phi\left(k+k^{\prime}\right)=\phi(k)+$ 
$\phi\left(k^{\prime}\right)$ and $\phi(r k)=r \phi(k)$, for all $k, k^{\prime} \in K$ and $r \in R$, follows trivially. We deduce that $K$ and $K k_{1}=\operatorname{Im}(\phi)$ are $R$-isomorphic.

If $K k_{1} \subset K$, we can repeat the process with $K$ replaced by $K k_{1}$ and obtain an $R$-module $K k_{2} k_{1} \subseteq K k_{1}$ which is $R$-isomorphic to $K k_{1}$ (and hence to $K$ ). And so we can continue to repeat this process until the containment is not proper any more (because of the $D C C R$ ) and we end up with a chain of $R$-subgroups:

$$
K \supset K k_{1} \supset K k_{2} k_{1} \supset \cdots \supset K k_{i} k_{i-1} \ldots k_{1}=K k_{i+1} k_{i} \ldots k_{1} \text {. }
$$

This implies that $k_{i+1} k_{i} \ldots k_{1}=k^{\prime} k_{i+1} k_{i} \ldots k_{1}$ for some $k^{\prime} \in K$, whence $R k_{i+1} k_{i} \ldots k_{1}=$ $R k^{\prime} k_{i+1} k_{i} \ldots k_{1} \subseteq K k_{i+1} k_{i} \ldots k_{1} \subseteq R k_{i+1} k_{i} \ldots k_{1}$ and it follows that $K k_{i+1} k_{i} \ldots k_{1}$ is monogenic over $R$ by $k_{i+1} k_{i} \ldots k_{1}$. Since all the subgroups in the chain are $R$-isomorphic, ${ }_{R} K$ is also monogenic.

Corollary 3.3. If $R$ has DCCR and contains a simple faithful $R$-subgroup, then $R$ is Oprimitive.

Note that Theorem 3.2 is no longer valid if ${ }_{R} K$ is not faithful: Let, for example, $G:=\mathbb{Z}_{2} \oplus \mathbb{Z}_{2} \oplus \mathbb{Z}_{2} \quad$ and let $H_{1}:=\{(0,0,0),(0,1,0)\}, \quad H_{2}:=\{(0,0,0),(1,0,0)\}, \quad H_{3}:=$ $\{(0,0,0)(1,1,0)\}$ and $H:=\sum_{i=1}^{3} H_{i}$. Then define $R$ as follows:

$$
R:=\left\{f \in M_{0}(G) \mid f\left(H_{i}\right) \subseteq H_{i} \text { for all } i=1,2,3\right\} .
$$

$R$ is a finite near-ring with identity. If we now take

$$
K=\{f \in R \mid f(0,0,1) \in H \text { and } f(\alpha)=(0,0,0) \text { for all } \alpha \neq(0,0,1)\},
$$

then it is easy to verify that ${ }_{R} K$ is simple, not faithful and also not monogenic.

Theorem 3.4. Suppose $R$ is finite. Then $\mathbb{M}_{n}(R)$ is O-primitive if and only if $R$ is O-primitive.

Proof. If $R$ is 0 -primitive then $\mathbb{M}_{n}(R)$ is 0-primitive by Lemma 1.8. Now suppose $M_{,}(R) \Gamma$ is a faithful type 0 module with generator $\gamma$. Then $\Gamma \cong \mathbb{M}_{n}(R) / \mathscr{L}$ as $\mathbb{M}_{n}(R)$-modules where $\mathscr{L}:=\operatorname{Ann}_{\mathbf{M}_{n}(R)}(\gamma)$ is a maximal left ideal of $\mathbb{M}_{n}(R)$. Since $M_{c}(R) \mathbb{M}_{n}(R) / \mathscr{L}$ is faithful, $\mathscr{L}$ cannot contain any two-sided ideals other than $\{0\}$. Also, since $\mathbb{M}_{n}(R)$ is finite, it contains minimal left ideals as well as minimal two-sided ideals. Suppose all minimal left ideals of $\mathbb{M}_{n}(R)$ are contained in $\mathscr{L}$. According to Pilz [9, 3.54], every minimal two-sided ideal is a direct sum of minimal left ideals. This would mean that $\mathscr{L}$ contains all the minimal two-sided ideals, which is impossible.

Consequently, there is at least one minimal left ideal, say $\mathscr{B}$, of $\mathbb{M}_{n}(R)$ such that $\mathscr{B} \nsubseteq \mathscr{L}$. Hence, $\mathscr{B} \gamma \neq\{0\}$. From Pilz [9, 3.10], it follows that $\mathscr{B} \cong \Gamma$ as $\mathbb{M}_{n}(R)$-modules.

Furthermore, since $\mathscr{B} \neq\{0\}$, there is a non-zero $\alpha \in R^{n}$ such that $\mathscr{B} \alpha$ is a non-zero $\mathbb{M}_{n}(R)$-subgroup of $R^{n}$. This implies that $\mathscr{B} \alpha$ is of the form $K^{n}$ for some non-zero $R$-subgroup $K$ of $R$. (Take $K=\left\{\pi_{1} B \alpha \mid B \in \mathscr{B}\right\}$.) The map $\mathscr{B} \rightarrow K^{n}$ which sends $B \in \mathscr{B}$ to $B \alpha$ for all $B \in \mathscr{B}$ assures us of an isomorphism 


$$
K^{n} \cong \mathscr{B} /\left(\mathscr{B} \cap \operatorname{Ann}_{M_{a}(R)}(\alpha)\right)=\mathscr{B} /\{0\} \cong \mathscr{B}
$$

of $\mathbb{M}_{n}(R)$-modules. Consequently, $\Gamma \cong K^{n}$ as $\mathbb{M}_{n}(R)$-modules whence $M_{\text {, }(R)} K^{n}$ is simple and faithful. We therefore must have ${ }_{R} K$ simple and faithful, by Lemma 3.1. Corollary 3.3 now implies that $R$ is 0 -primitive.

Corollary 3.5. If $R$ is a finite O-primitive near-ring, then there exist a maximal left ideal $\mathscr{L}$ and a minimal left ideal $\mathscr{B}$ of $\mathbb{M}_{n}(R)$ such that

$$
\mathbb{M}_{n}(R)=\mathscr{L} \oplus \mathscr{B}
$$

Proof. Following the same terminology as in the proof of Theorem $3.4, \mathscr{B} \cap \mathscr{L}=\{0\}$ by the minimality of $\mathscr{B}$ and we therefore must have that $\mathbb{M}_{n}(R)=\mathscr{L} \oplus \mathscr{B}$, by the maximality of $\mathscr{L}$.

The following corollary clears up - at least to a certain extent-open problem 5 posed in Meyer [7, p. 105]. For any $k, 1 \leqq k \leqq n, \mathscr{L}_{k}$ is defined to be the left ideal of $\mathbb{M}_{n}(R)$ generated by the matrix $f_{1 k}^{1}$. We also define

$$
\mathscr{M}_{k}:=\mathscr{L}_{1}+\mathscr{L}_{2}+\cdots+\mathscr{L}_{k-1}+\mathscr{L}_{k+1}+\cdots+\mathscr{L}_{n}
$$

In Meyer [6] it is shown that if $F$ is a near-field, then, with $R$ replaced by $F$ in the foregoing, $\mathscr{M}_{k}$ is a maximal left ideal of $\mathbb{M}_{n}(F)$. Moreover, it is shown that

$$
\mathscr{M}_{k}=\operatorname{Ann}_{\mathrm{M}_{k}(F)}\left(l_{k}(1)\right)
$$

Corollary 3.6. If $F$ is a finite near-field and with the notation as explained above, there is a minimal left ideal $\mathscr{B}$ of $\mathbb{M}_{n}(F)$ such that $\mathscr{B} \cap \mathscr{M}_{k}=\{0\}$ and hence that

$$
\mathbb{M}_{n}(F)=\mathscr{M}_{k} \oplus \mathscr{B}
$$

Proof. The module ${ }_{M_{a}(F)} F^{n}$ is faithful and of type 0 and we may choose $\gamma:=l_{k}(1)$ as generator. But, according to $(\dagger), \mathscr{M}_{k}$ is the annihilator of $\gamma$ in the near-ring $\mathbb{M}_{n}(F)$. Following the proofs of Theorem 3.4 and Corollary 3.5 above, our result is immediate.

It remains, however, to be seen whether $\mathscr{B} \subseteq \mathscr{L}_{k}$ in the corollary above, as was suggested by the open problem discussed in the foregoing.

Another question which remains open is whether Lemma 1.10 remains valid if $\Gamma$ is a type $0 \mathbb{M}_{n}(R)$-module. Examples suggest very strongly (at least in the finite case) that this is indeed the case. This would in turn, force Lemma 1.11 to be true in the 0 primitive case and by using Theorem 1.7 one should be able to prove a strong link between $\mathscr{T}_{0}(R)$ and $\mathscr{T}_{0}\left(\mathbb{M}_{n}(R)\right)$ which we formalise as follows: 
Conjecture 3.7. If $R$ is finite, then

$$
\mathscr{T}_{0}\left(\mathbb{M}_{n}(R)\right)=\left(\mathscr{T}_{0}(R)\right)^{*}
$$

Acknowledgements. This paper was written while the author was visiting the Department of Mathematics at the University of Edinburgh, Scotland. He was financially supported by the CSIR, the Central Research Fund of the UOFS and his parents. He wishes to express his appreciation towards these institutions and in particular to his parents. He would also like to thank Dr John Meldrum and his family for their hospitality and assistance during his visit.

\section{REFERENCES}

1. S. J. AbBasI, Matrix near-rings and generalized distributivity (Doctoral dissertation, University of Edinburgh, Edinburgh, Scotland, 1989).

2. S. J. Abbasi, J. D. P. Meldrum and J. H. Meyer, The $\mathscr{T}_{0}$-radical of matrix near-rings, Arch. Math., to appear.

3. S. J. Abbasi, J. D. P. Meldrum and J. H. Meyer, Ideals in near-rings and matrix near-rings, submitted.

4. J. D. P. Meldrum, Near-rings and their links with groups (Research Notes in Mathematics 134, Pitman, London, 1985).

5. J. D. P. Meldrum and A. P. J. Van der Walt, Matrix near-rings, Arch. Math. 47 (1986), 312-319.

6. J. H. Meyer, Left ideals in matrix near-rings, Comm. Algebra 17 (1989), 1315-1335.

7. J. H. Meyer, Matrix near-rings (Doctoral dissertation, University of Stellenbosch, Stellenbosch, South Africa, 1986).

8. J. H. Meyer and A. P. J. VAN der W WLt, Solution to an open problem concerning 2-primitive near-rings, in Near-rings and Near-fields (ed. G. Betsch, North-Holland, 1987), $185-192$

9. G. Pilz, Near-rings (Revised edition, North-Holland, 1983).

10. D. R. Stone, Maximal left ideals and idealizers in matrix rings, Canad. J. Math. 32 (1980), $1397-1410$.

11. A. P. J. VAN der Walt, Primitivity in matrix near-rings, Quaestiones Math. 9 (1986), 459-469.

12. A. P. J. VAN DER WALT, On two-sided ideals in matrix near-rings, in Near-rings and Near-fields (ed. G. Betsch, North-Holland, 1987), 267-272.

13. A. P. J. VAN DeR $W_{A L T}$ and $L$. VAN WYK, The $\mathscr{T}_{2}$-radical in structural matrix near-rings, $J$. Algebra 123 (1989), 248-261.

Department of Mathematics

University of the Orange Free State

P.O. Box 339

BLOEMFONTEIN

9300

Republic of South Africa 